\title{
Jet Impingement Boiling Heat Transfer-A Complex and Effective Phenomenon
}

\author{
Mohamed Hamed \\ Thermal Processing Laboratory, Department of Mechanical Engineering \\ McMaster University \\ Hamilton, Ontario, Canada
}

Accelerated cooling is essential for various technological applications such as electronic cooling, thermal processing of materials, and nuclear cooling systems. Jet Impingement Boiling (JIB) offers significantly high cooling rates and involves a complicated fluid flow and heat transfer problem. The high cooling rates provided by JIB are due to the effective interplay of various modes of boiling heat transfer and jet hydrodynamics. This talk provides an overview of the complicated fluid flow-heat transfer phenomena associated with JIB and a discussion of the various approaches used in modelling JIB, including the effort of our research team at McMaster Thermal Processing Laboratory (TPL). 